\title{
Inhalers and nebulizers: basic principles and preliminary measurements
}

\author{
Ondrej Misik ${ }^{1 *}$, Frantisek Lizal ${ }^{1}$, Vahid Farhikhteh Asl ${ }^{2}$, Miloslav Belka ${ }^{1}$, Jan Jedelsky ${ }^{1}, J_{a k u b}$ Elcner $^{1}$ and Miroslav Jicha ${ }^{1}$ \\ ${ }^{1}$ Brno University of Technology, Faculty of Mechanical Engineering, Energy Institute, Technicka 2896/2, Brno 616 69, Czech Republic \\ ${ }^{2}$ Dresden University of Technology, Faculty of Environmental Science, Urban and Industrial Water Management Institute, Bergstraße 66 , \\ 01069 Dresden, Germany
}

\begin{abstract}
Inhalers are hand-held devices which are used for administration of therapeutic aerosols via inhalation. Nebulizers are larger devices serving for home and hospital care using inhaled medication. This contribution describes the basic principles of dispersion of aerosol particles used in various types of inhalers and nebulizers, and lists the basic physical mechanisms contributing to the deposition of inhaled particles in the human airways. The second part of this article presents experimental setup, methodology and preliminary results of particle size distributions produced by several selected inhalers and nebulizers.
\end{abstract}

\section{Introduction}

The inhalation treatment becomes very popular nowadays. It is one of the most effective non-invasive way of the treatment among the large range of diseases from cancer to migraines [1]. The effect of the inhalation therapy depends on rate and place of particle deposition in the airway. There are three essential mechanisms of particle deposition in lungs: inertial impaction, gravitational sedimentation and Brownian diffusion. The largest particles, mostly larger than $5 \mu \mathrm{m}$, are deposited by inertial impaction particularly in upper airways. The Stokes number plays an essential role in this mechanism of deposition [2;3]. Then, in the tracheobronchial tree, the airstream is decelerated as the total area of the crosssection increases. It causes that particles reaching this part of the lungs are settled down by gravitational sedimentation. Particles with size between $1 \mu \mathrm{m}$ and $5 \mu \mathrm{m}$ are usually deposited by this mechanism. Particles in sizes of $0.1-1 \mu \mathrm{m}$ are mostly exhaled from lungs, but particles smaller than $100 \mathrm{~nm}$ are deposited well with the same probability in all parts of the lung due to the diffusion $[1 ; 2 ; 4]$. Generally, it is not necessary to deposit the particles in the alveolar regions, where absorption of drug to the blood circulation is highest. Particularly in the case of lung diseases, the demanded deposition is in the regions, where the receptors for wanted effect are located. The targeted deposition of drugs is a task for inhalation systems [5].

Generally, the current inhalation systems can be divided to three main categories: nebulizers, metered dose inhalers (MDI) and dry powder inhalers (DPI). The background of the inhalation treatment originates from antiquity. First reference of inhalation therapy was the papyrus from ancient Egypt in 1554 BC. This papyrus

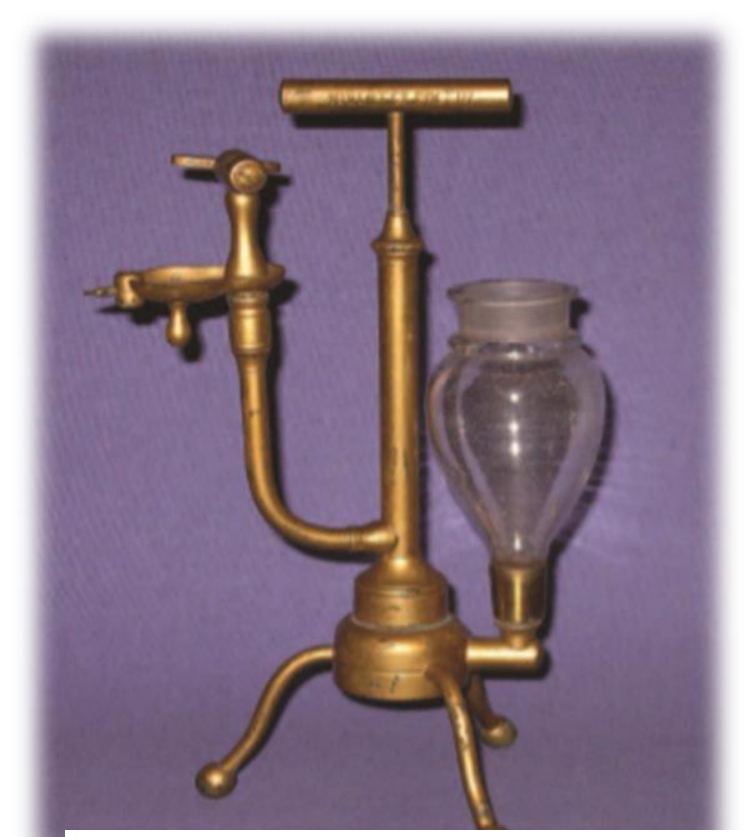

Fig. 1. The Sales-Girons nebulizer. [8]

\footnotetext{
*Corresponding author: misik.ondro@gmail.com
} 


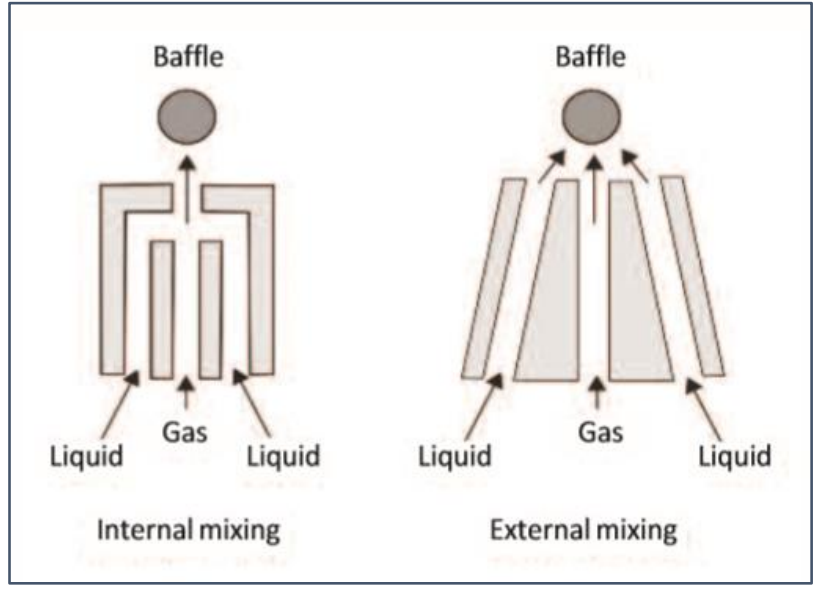

Fig. 2. Typical designs of the jet nebulizers. [8]

describes inhaling of the vapours created from the black henbane (Hyoscyamus niger) put onto hot bricks. Over the many years, there were a lot of improvised ways and devices for inhalation therapy. After the industrial revolution, the inhalation devices became mass produced. In the middle of $19^{\text {th }}$ century, inhalation was used for the first time to the anaesthesia. The first nebulizer (Sales-Girons nebuliser) was invented in 1858 as a replacement of spas for people who cannot afford it (Fig. 1). Smoking the asthma cigarettes and inhaling the smoke of burned powders was also very popular at that time. Inhalation devices in the current form had been developing during the $20^{\text {th }}$ century. The first DPI, Aerohaler, was designed in 1948 for inhaling the benzylpenicillin. MDIs are produced since 1956, when the Riker laboratories brought Medihaler to the market. MDIs were firstly manufactured with CFCs (Chlorofluorocarbons) formulation. However, the usage of CFCs was banned by Montreal Protocol in 1987 because of the ozone layer depletion and CFC was replaced by HFA (hydrofluoroalkane) [6;7].

The nebulizers are non-portable devices which nebulize a liquid drug solution. They require connection to the electric network during the usage. They are classified based on the technology into three categories: jet, ultrasound and mesh nebulizers. The principle of jet nebulizers is formed on the Venturi tube, where air from the compressor is accelerated through the jet and goes near the small capillary tube in which a negative pressure is created. In view of the fact that liquid is sucked up from a reservoir in contact with airstream, it is atomized because of shear forces. A baffle is placed in front of the airflow in order to catch the larger aerosol particles and return them back to the reservoir. The common designs of the jet nebulizers are shown on the Fig. 2 [5; 8]. For more economical usage were invented so called Breathenhanced inhalers (BEN) and Breath-actuated inhalers (BAN). Whereas the standard jet nebulizers generate aerosol constantly BEN and BAN try to reduce its aerosol production during the exhalation. The principle of the aerosol generation in BEN is the same as in the case of the standard jet nebulizer, but a one-way valve is located above the nebulizing chamber. Another one-way valve is located on the mouthpiece and the aerosol is

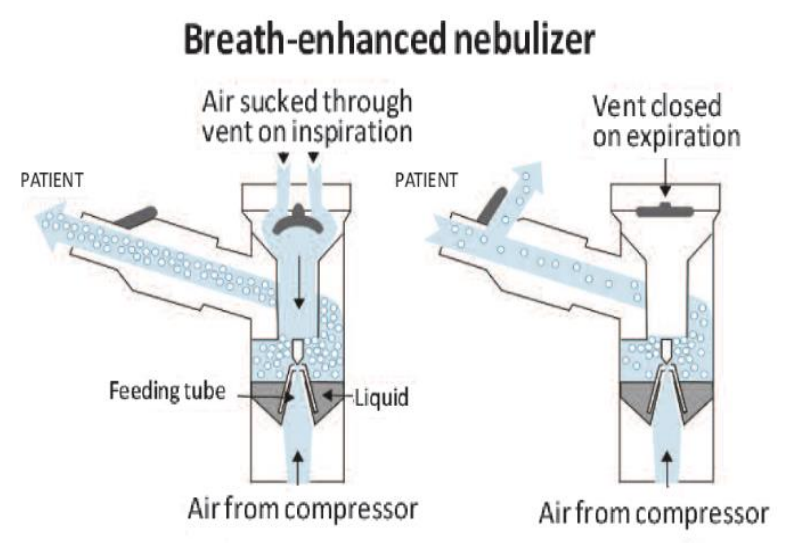

Fig. 3. The principle of the BEN. [8]

generated constantly in the nebulizing chamber. The valve above the nebulizing chamber is open during the inspiration which makes the aerosol to come out from the nebulizer. During the expiration, the first valve is closed and the valve on the mouthpiece is opened. This causes that the aerosol remains in the device during expiration and exhaled air is blown out through the valve on the mouthpiece (Fig. 3). BANs are often the nebulizers with electronic control which switch on during inspiration in order to decrease a wastage. For instance, the AeroEclipse nebulizer has a green button on the top of the device. This button moves down during the inhalation due to the negative pressure. The piston connected to the button is placed on the spring and the movement activates the aerosol production. During the exhalation, the piston moves back and switches off the device. The green button also signalizes to the patient that they are inhaling correctly. This nebuliser is suitable for old patients and children because of the high device sensitivity $[8 ; 9 ; 10]$. Principally, ultrasound nebulizers create a standing wave on a liquid, which produce an aerosol on the liquid surface. The vibrations heat up the solution, so this device is suitable for thermostable drugs only $[5 ; 10]$. In the mesh nebulizers, the liquid drug solution is forced through a vibrating mesh with a number of little orifices producing an aerosol. Although

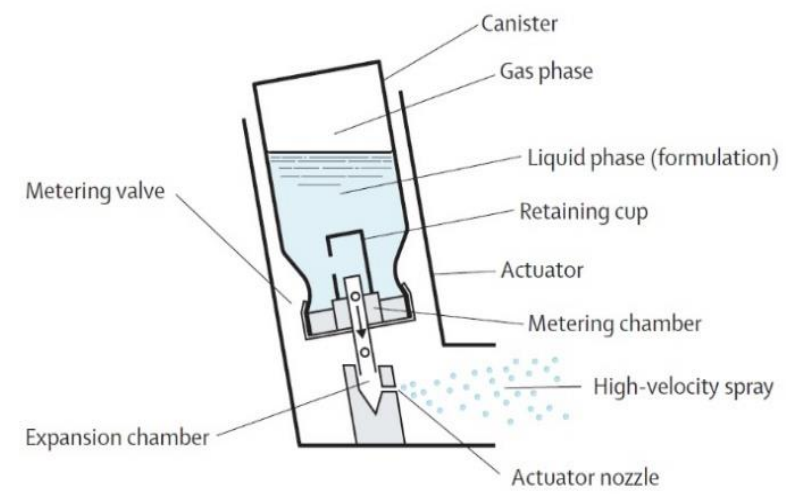

Fig. 4. The scheme of the usual MDI. [21] 
the mesh nebulizers are the most effective, they are also the most expensive and the most complicated $[5 ; 10]$.

Another big group of inhalers are metered dose inhalers (MDI) which have been popular for a long time because they are small, portable and easy to use. General components of the MDI are a canister, metering valves including inner and outer one, and an actuator mouthpiece as you can see on the Fig. 4 [11;5]. The canister is filled with solution of the propellant, drug, surfactant and some other constituents. The initial state before usage of the MDI occurs when the inner valve is open and the outer one is closed. During the usage, the inner valve is closed to meter the precise dose of the formulation, subsequently the outer one is allowed to open and emits the aerosol, and the aforementioned cycle repeats in the same way [11]. A coordination between actuation of the device and inspiration causes many complications. This was solved by an invention of a breath-actuated MDI, such as Autohaler. This inhaler contains a compressed spring, which causes actuation of the device when the jet is opened by the inspiration [5]. Owing to the propellant usage, velocity of the emitted aerosol is high which causes significant oropharyngeal deposition. That is the reason to use additional devices, such as spacers or chambers. These components prolong the distance between the mouthpiece and the mouth cavity which slows down the aerosol flow. Furthermore, it eliminates the problem with coordination of the inspiration and actuation [12].

The usage of propellants is non-ecological. Moreover, high velocity causes complications with coordination. Avoiding that was the aim of invention of a Soft Mist Inhalers (SMI), such as Respimat. These devices are light-weight portable devices which nebulize the liquid drug solution using mechanical energy of spring and emit the metered dose of soft mist. When the patient rotates the inhaler body by $180^{\circ}$, the cartridge from the reservoir is loaded to a metering chamber. The gear transforms a rotation into a linear movement and compresses the spring. By clicking the dose-release button, the spring is released which forces the dose through a tiny twin-nozzle in a uniblock part, where nozzles are at the controlled angle. It makes the two streams collide and produces a soft mist with low velocity $[13 ; 5 ; 14]$.

The last category of inhalers is dry powder inhalers (DPI) which have three divisions: Single-dose, Multiunit-dose and Multi-dose inhalers [15]. Single-dose

Table 1. The comparison of the resistance and the airflow during the inspiration in selected devices

\begin{tabular}{|c|c|c|}
\hline Inhalation devices & $\begin{array}{c}\text { Resistance of } \\
\text { the device } \\
{\left[\mathrm{kPa}^{0,5} \mathrm{l} / \mathrm{min}\right]}\end{array}$ & $\begin{array}{c}\text { Flow during } \\
\text { inspiration } \\
\text { [I/min] }\end{array}$ \\
\hline Breezhaler ${ }^{\circledR}$ & 0,017 & 111 \\
\hline Aerolizer® & 0,019 & 102 \\
\hline Elliptaß & 0,027 & 74 \\
\hline Novolizer® & 0,027 & 72 \\
\hline Accuhaler/Diskus ${ }^{\circledR}$ & 0,027 & 72 \\
\hline Genuair $\circledR$ & 0,031 & 64 \\
\hline Nexthaler® & 0,036 & 54 \\
\hline Turbohaler® & 0,039 & 54 \\
\hline Handihaler ${ }^{\circledR}$ & 0,058 & 37 \\
\hline
\end{tabular}

inhalers have metered dose of drug powder packed into a capsule. Prior to an inhalation, the capsule is set into a device and pierced to release the dose. The Multiunitdose inhalers encompass plenty doses in one blister, so there are many doses available. The Multi-dose inhaler possesses a reservoir with powder formulation and it meters a precise dose of powder before the inhalation [15]. The powder is influenced by three types of forces: the interparticle force, the dispersion force created by a device and the force of the inspiration. The aim is to keep these forces in balance, which means the balance between resistance of the device and airflow of inspiration. The comparison of the selected DPI is shown in Tab 1. Strong airflow results in the deposition in the oropharyngeal region, whereas the higher device resistance makes it difficult to inhale for children, old people or people with advanced-stage diseases. So, we can sort devices also by their resistance as low resistance, medium resistance and high resistance devices $[16 ; 17$; 5]. Another important point is deagglomeration of particle clusters. As was mentioned above, the interparticle forces act between the particles creating particle agglomerates. Particles should be deagglomerated during inhalation. For this purpose, a mesh can be set into the airflow or the mouthpiece geometry can be shaped to create whirls and turbulent flows. A humidity causes huge problems for deagglomeration, hence the DPI should be stored on a dry place and patient must not exhale into the device [5].

Experimental measurements of particle size distributions coming from various kinds of inhalers were performed. The obtained data were used to estimate lung deposition.

\section{Experimental measurement}

The experimental measurements which are described in this part are based on Misik's bachelor thesis Inhalers and nebulizers for medical use [5]. Particle size distributions of selected devices were measured and their FPF (Fine Particle Fraction) were evaluated. FPF represents the percentage of particles which deposit well in the lung, i.e.

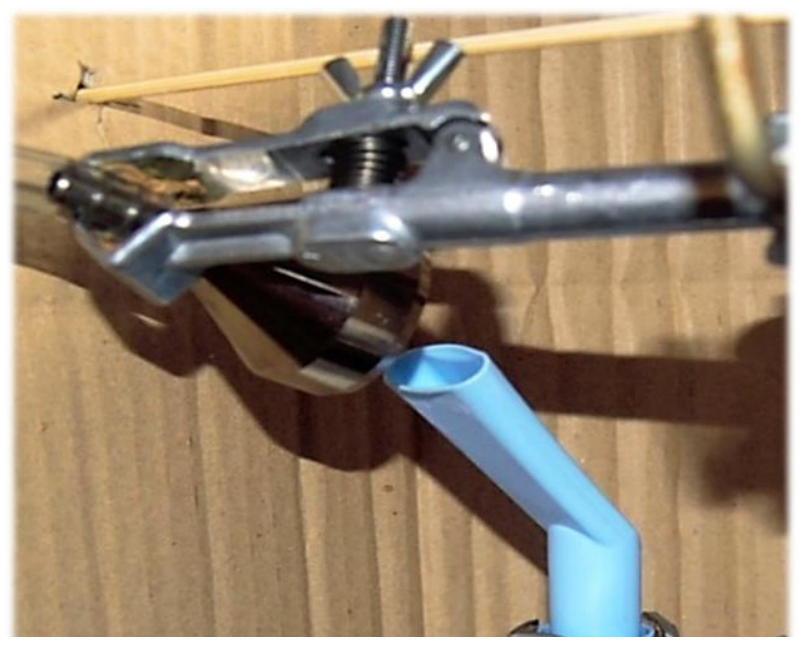

Fig. 5. The measurement assembly of the InnoSpire nebulizer. [5] 
particles in the range $1-5 \mu \mathrm{m}$. Measurements were done using the Aerodynamic Particle Sizer (APS, TSI 3321, TSI Inc., Shoreview, MN, USA), which measures an aerodynamic particle diameter. The inhaler was connected to the APS via a conductive tube to eliminate deposition on the walls of the tube because of the electrostatic force. An isokinetic probe was connected at the inlet of the tube to eliminate the effect of a stream angle and velocity differences. Nebulizers and MDI were sampled in the open air because sampling into the closed box results in coagulation of particles. In addition, the nebulizer particles were coagulating in open space due to constant generation of aerosol for quite a long time [5]. In terms of DPI, it was necessary to simulate an inspiration, therefore the DPIs were sampled into a plastic canister connected to a simulator of breathing. Since the canister was not air-tight, it was necessary to induce an inspiration flow of $1.5 \mathrm{l} / \mathrm{s}$ on the simulator. In general, measurement was more precise when the distance between the mouthpiece and isokinetic probe was short [5]. Following devices were measured: nebulizer InnoSpire ${ }^{\circledR}$ Elegance (Philips), metered dose inhaler Atrovent ${ }^{\circledR}$ (Boehringer Ingelheim), soft mist inhaler Respimat ${ }^{\circledR}$ (Boehringer Ingelheim) and dry powder inhalers Ellipta ${ }^{\circledR}$ (Glaxosmithkline) and Breezhaler ${ }^{\circledR}$ (Novartis) [5].

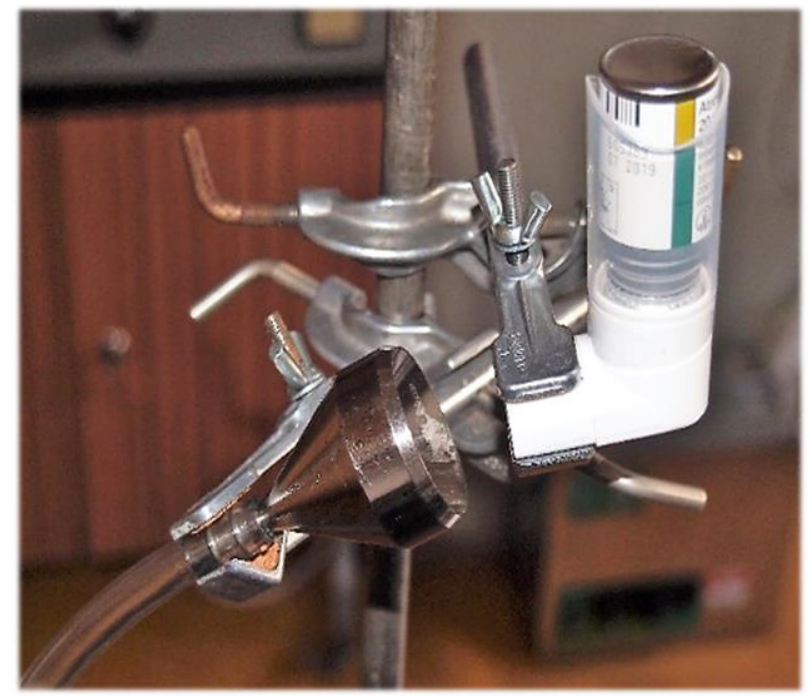

Fig. 6. The measuring assembly of the Atrovent MDI. [5]

\subsection{InnoSpire ${ }^{\circledR}$ Elegance}

During the measurement, the nebulization chamber was connected to a compressor with maximum pressure $317 \mathrm{kPa}$ and airflow of $6 \mathrm{l} / \mathrm{min}$. The chamber was filled with distilled water and oriented in the vertical direction. The hose connected to the APS had to be bended, what might have caused deposition in the hose by impaction. Since the aerosol flow from nebulizers is quite low, the direction of flow is susceptible to external influences like airstreams in the laboratory. To prevent these effects, it was required to make a partial cover which had to be open to avoid coagulation. The photo of the measuring assembly of InnoSpire nebulizer can be seen on the Fig. 5 [5].

\subsection{Atrovent $^{\circledR}$}

During the measurement of the Atrovent inhaler, an effort was made to reach a constant flow of aerosol, so several doses were emitted during one sample. Similar procedure was done in the measurement of particle size distribution of the Respimat ${ }^{\circledR}$ SMI. Each time, the device had to be actuated manually which might have caused small movements of the device during the sampling. The MDI emits the aerosol with high velocity so small movements could have caused variability in results. The photo of the experiment with Atrovent is on the Fig. 6 [5].

\subsection{Dry Powder Inhalers}

As was mentioned previously, it was necessary to use the canister and the simulator of breathing for sampling during the measurement of dry powder inhalers. Another complication was that any effort to provide the continual sampling was unsuccessful. Therefore, the sampling time was shortened to $4 \mathrm{~s}$. The mean diameter decreased during the sample time, as only particles with small diameter remained in the canister. To sum up, these measurements were least accurate [5].

\section{Results and discussion}

Table 2. Fine particle fractions and CMADs of measured inhalers. [5]

\begin{tabular}{lll} 
Inhalation device & FPF [\%] & CMAD $[\boldsymbol{\mu m}]$ \\
\hline \hline InnoSpire $^{\circledR}$ Elegance & $75,05 \pm 5,09$ & $3,66 \pm 0,22$ \\
Atrovent ${ }^{\circledR} 1$. & $59,37 \pm 6,93$ & $1,08 \pm 0,06$ \\
Atrovent $^{\circledR}$ 2. & $69,30 \pm 4,26$ & $1,20 \pm 0,05$ \\
Atrovent $^{\circledR}$ 3. & $78,59 \pm 0,89$ & $1,29 \pm 0,01$ \\
Respimat $^{\circledR}$ SMI Placebo & $58,74 \pm 3,65$ & $3,94 \pm 0,25$ \\
Respimat $^{\circledR}$ SMI Spiolto & $56,67 \pm 4,23$ & $4,30 \pm 0,27$ \\
Breezehaler $^{\circledR}$ & $83,16 \pm 2,51$ & $2,80 \pm 0,18$ \\
Brimica $^{\circledR}$ Genuair $^{\circledR}$ & $66,21 \pm 5,66$ & $1,31 \pm 0,131$ \\
ANORO $^{\circledR}$ Ellipta $^{\circledR}$ & $80,09 \pm 0,95$ & $1,85 \pm 0,10$ \\
\hline
\end{tabular}

In data analyses FPF and count median of aerodynamic diameter (CMAD) were calculated. In our case the

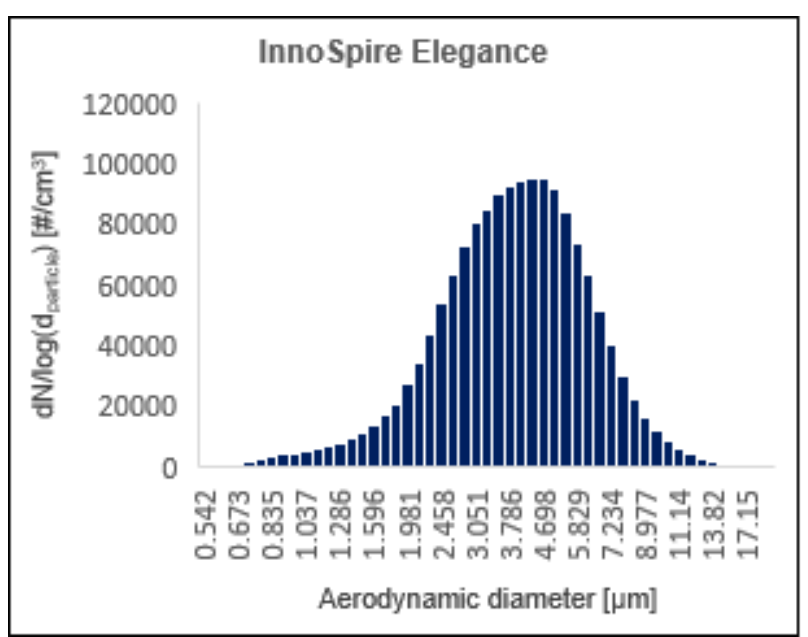

Fig. 7. The measured particle size distribution of the InnoSpire nebulizer. [5] 


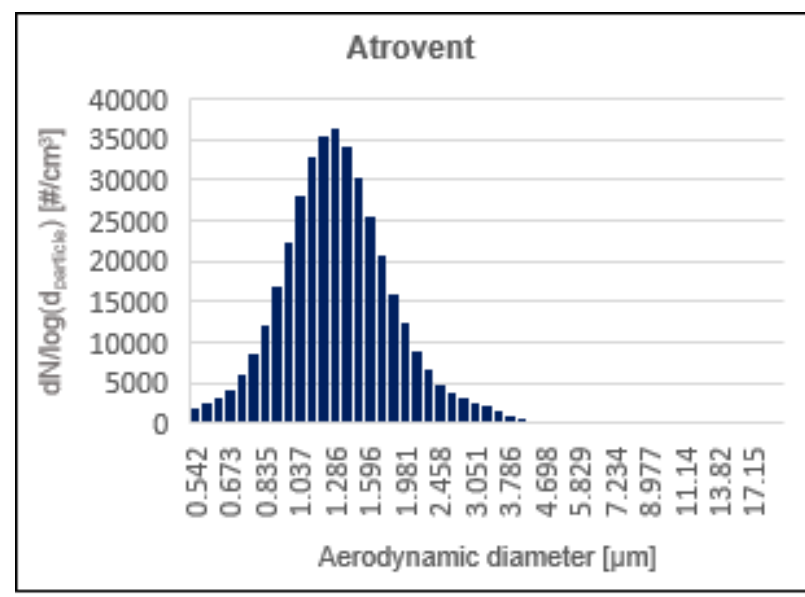

Fig. 8. The measured particle size distribution of the Atrovent MDI [5].

calculated FPF was represented by particles in the range of $1-4.7 \mu \mathrm{m}$ [5]. The obtained data are shown in the Tab 2.

The technical description of the InnoSpire device declares that its FPF calculated as a fraction of particles $<5 \mu \mathrm{m}$ is $77 \%$ [18]. In our measurements, the average FPF of this nebulizer was about $75 \%$. Therefore, very good agreement was found. However, from obtained data we can see that particles coagulated considerably. The nebulizer was turned on for approximately 2.5 minutes and the measurements were done in a sequence without long pauses. Based on this data, it can be seen that during the experiment, on one hand, the number of small particles was decreasing and on the other hand, the amount of the large particles was increasing. At the beginning, the FPF of this device was about $65 \%$ and it grew till the end of the experiment, when it reached about $80 \%$. So, in this case the coagulation had a positive impact. However, the inhalation therapy with this device should last $6-8$ minutes and the results can be different. For future measurement, it would be suitable to simulate a breathing pattern during the experiment to check up the coagulation in the case of real flow in the mouthpiece. The measured size distribution of InnoSpire nebulizer is shown on the Fig. 7 [5].

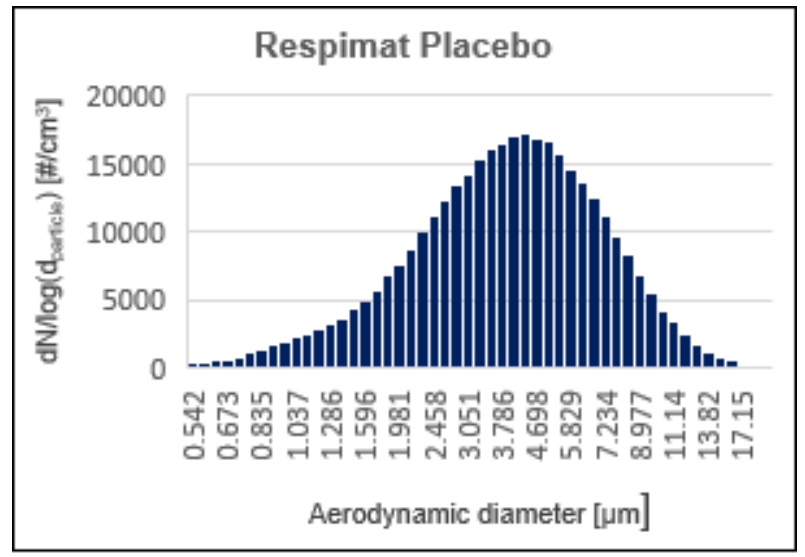

Fig. 9. The measured particle size distribution of the Respimat Placebo SMI [5].

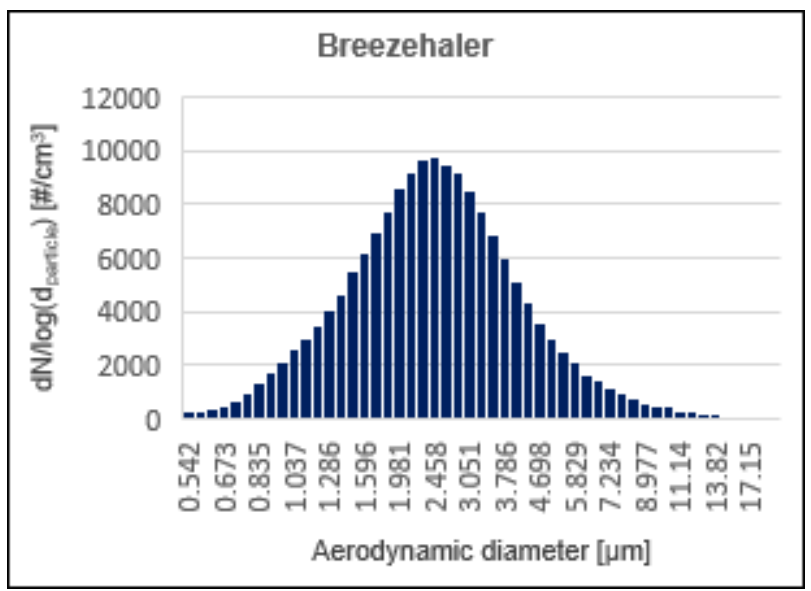

Fig. 10. The measured particle size distribution of Breezehaler DPI [5].

In the data from measurement of the Atrovent particle size distribution, a big variability was observed between each set of data. Because of that, three different sets of the measurements were done (Atrovent 1., Atrovent 2. Atrovent 3.). There were small changes in the assembly setting (like distance and angle between isokinetic probe and the mouthpiece of the device) between each set; and each set consists at least from 4 measurements. As was already mentioned, the manual actuation of the device was performed which could cause small movements of the device. This probably caused the variability of aerosol velocity, the movements and variable direction of mouthpiece could have a considerable impact on the results. There was also a different distance between the isokinetic probe and the mouthpiece in each measurement. Mitchel et al. [19] measured the particle size distribution with Aerosizer. In their experiment, the FPF was about $97 \%$ and representing the particles $<4.6 \mu \mathrm{m}$, while in the case that particles smaller than $1 \mu \mathrm{m}$ were added to calculation, the obtained values were about $99 \%$, which is in agreement with the data from Aerosizer. The measured particle size distribution can be seen on the Fig. 8 [5].

Results of both Respimat SMI formulations were nearly identical. Respimat SMI is developed to create particles smaller than $5.8 \mu \mathrm{m}$ and FPF should range between $65-80 \%$. In this case, particle fraction calculated with range of particles $<5.8 \mu$ m was $77 \%$ for Placebo and $73 \%$ for Spiolto formulation. In this case the different formulation had no influence on the particle size distributions (Fig. 9) [5].

As mentioned before, there was a lot of inaccuracies in the measurements of the DPI. The canister was not air tight, because of the imperfect sealing in the places of the hose connection. Another problem of the sampling was the inability of the simulator to stop the cycle after the inspiration. Inspiration sucked the powder from the device and then the flow induced by simulator during expiration disturbed the flow inside canister and blowed out the particle from the isokinetic probe. Lastly, the measured distributions gave us an overview of DPI particle sizes. The measured particle size distribution is shown on the Fig. 10 [5]. 


\section{Conclusions}

In this paper, the preliminary results of the particle size distributions from several selected inhalers were presented and practical issues about accuracy of the measurements were discussed.

In the InnoSpire Elegance nebulizer, the coagulation of the particles was detected. In the future measurement, it would be appropriate to investigate if the mouthpiece is the reason of the coagulation and if the particles are coagulating also in case of a cyclic breathing regime [5].

MDI Atrovent showed significant variability of the results. It was attributed to the high velocity of the aerosol stream and instability of the device during the measurement. This could be solved by using the spacer to enlarge the distance between the mouthpiece and the isokinetic probe and slow down the aerosol before entering the airways. To reduce the complications with the non-continual dosing during the sampling time, it could be useful to perform the measurements with the Cascade Impactor where the sampling time is not relevant. The ipratropium bromide is the effective component of the Atrovent formulation which makes the maximal bronchodilatation effect when MMAD (mass median of aerodynamic diameter) of the aerosol is about $3 \mu \mathrm{m}$ [20]. MMADs calculated from CMADs specified in the table of the results were between $1.6-2.0 \mu \mathrm{m}$. It is probable that after the application of the spacer and after solving the problem of non-continual dosing, MMAD will increase and approach values about $3 \mu \mathrm{m}$ [5].

The measurements of DPI bring information about particle size distribution, but the results were influenced by a few deficiencies, where the most important one was probably that the device was able to release just one dose during the sampling time. Because of this, the future measurement should include also the Cascade Impactor to avoid the influence of the sampling time [5].

\section{Acknowledgement}

This work was supported by the Czech Science Foundation under the grant GA16-23675S, by the project FSI-S-17-4444, and by the project LTC17087 of the programme INTER-EXCELLENCE, sub-programme INTER-COST.

\section{References}

1. J. Patton and P. Byron, Nature Reviews Drug Discovery vol. 6, 67-74, (2007).

2. W. Hinds, Aerosol technology: properties, behavior, and measurement of airborne particles, 2nd ed. (1999).

3. C. Loira-Pastoriza, J. Todoroff and R. Vanbever, Advanced Drug Delivery Reviews vol. 75, 81-91, (2014).

4. A. Fernández Tena and P. Casan Clarà, Archivos de Bronconeumología (English Edition) vol. 48, 240246, (2012).
5. O. Mišík, Bachelor thesis, Brno University of Technology, (2017).

6. S. Stein, Journal of Aerosol Medicine and Pulmonary Drug Delivery vol. 30, 20-41, (2016).

7. P. J Anderson MD, Respiratory Care, vol. 50, 1139 1150, (2005).

8. O. Nerbrink, Inhalation, vol. 10, 9-14, (2016).

9. K. Leung, E. Louca and A. Coates, Chest, vol. 126, 1619-1629, (2004).

10. A. Ari, Eurasian Journal of Pulmonology, vol. 16, 1-7, (2014).

11. S. Stein, P. Sheth, P. Hodson and P. Myrdal, AAPS PharmSciTech vol. 15, 326-338 (2014).

12. S. Newman, Respiratory Care vol. 50, 1177-1190 (2005).

13. R. Dalby, J. Eicher and B. Zierenberg, Medical Devices: Evidence and Research 2011, 4, 145-155 (2011).

14. V. Kašák and E. Feketeová, Farmakoterapie vol. 6 4, (2010).

15. L. Garcia-Contreras, M. Ibrahim and R. Verma, Medical Devices: Evidence and Research 2015, 8, (2015).

16. R. Dal Negro, Multidisciplinary Respiratory Medicine vol. 10, 1, 1-4 (2015).

17. M. Hoppentocht, P. Hagedoorn, H. Frijlink and A. de Boer, Advanced Drug Delivery Reviews vol. 75, 18-31 (2014).

18. R. Hatley, R. Potter and Y. Dagtyareva, (n.d.), (2016)

19. J. Mitchell, M. Nagel and Y. Chengà, J. Aerosol Sci. 30, (1999).

20. P. Zanen, L. Go and J. Lammers, Thorax vol. 51, 977-980 (1996).

21. M. Dolovich and R. Dhand, The Lancet vol. 377, 1032-1045 (2011). 